University of Wollongong

Research Online

Faculty of Engineering and Information

Faculty of Engineering and Information

Sciences - Papers: Part B

Sciences

2020

Attention-Based Knowledge Tracing with Heterogeneous Information

Network Embedding

Nan Zhang

Ye Du

Ke Deng

$\mathrm{Li} \mathrm{Li}$

Jun Shen

University of Wollongong, jshen@uow.edu.au

See next page for additional authors

Follow this and additional works at: https://ro.uow.edu.au/eispapers1

Part of the Engineering Commons, and the Science and Technology Studies Commons

Research Online is the open access institutional repository for the University of Wollongong. For further information contact the UOW Library: research-pubs@uow.edu.au 


\title{
Attention-Based Knowledge Tracing with Heterogeneous Information Network Embedding
}

\author{
Abstract \\ Knowledge tracing is a key area of research contributing to personalized education. In recent times, deep \\ knowledge tracing has achieved great success. However, the sparsity of students' practice data still limits \\ the performance and application of knowledge tracing. An additional complication is that the contribution \\ of the answer record to the current knowledge state is different at each time step. To solve these \\ problems, we propose Attention-based Knowledge Tracing with Heterogeneous Information Network \\ Embedding (AKTHE). First, we describe questions and their attributes with a heterogeneous information \\ network and generate meaningful node embeddings. Second, we capture the relevance of historical data \\ to the current state by using attention mechanism. Experimental results on four benchmark datasets \\ verify the superiority of our method for knowledge tracing.
}

\section{Keywords}

information, heterogeneous, network, tracing, embedding, knowledge, attention-based

Disciplines

Engineering | Science and Technology Studies

\section{Publication Details}

Zhang, N., Du, Y., Deng, K., Li, L., Shen, J. \& Sun, G. (2020). Attention-Based Knowledge Tracing with Heterogeneous Information Network Embedding. Lecture Notes in Computer Science, 12274 95-103.

\section{Authors}

Nan Zhang, Ye Du, Ke Deng, Li Li, Jun Shen, and Geng Sun 


\title{
Attention-based Knowledge Tracing with Heterogeneous Information Network Embedding
}

\author{
Nan Zhang ${ }^{1}, \mathrm{Ye} \mathrm{Du}^{1}, \mathrm{Ke} \mathrm{Deng}^{2}, \mathrm{Li} \mathrm{Li}^{1 凶}{ }^{1} \mathrm{Jun}_{\mathrm{Shen}}^{3}$, and Geng $\mathrm{Sun}^{3}$ \\ 1 School of Computer and Information Science, Southwest University, Chongqing, \\ China \\ 2 RMIT University, Australia \\ 3 University of Wollongong, Australia \\ \{kathy525, duye99\}@email.swu.edu.cn, ke.deng@rmit.edu.au, \\ lily@swu.edu.cn, \{jshen, gsun\}@uow.edu.au
}

\begin{abstract}
Knowledge tracing is a key area of research contributing to personalized education. In recent times, deep knowledge tracing has achieved great success. However, the sparsity of students' practice data still limits the performance and application of knowledge tracing. An additional complication is that the contribution of the answer record to the current knowledge state is different at each time step. To solve these problems, we propose Attention-based Knowledge Tracing with Heterogeneous Information Network Embedding (AKTHE). First, we describe questions and their attributes with a heterogeneous information network and generate meaningful node embeddings. Second, we capture the relevance of historical data to the current state by using attention mechanism. Experimental results on four benchmark datasets verify the superiority of our method for knowledge tracing.
\end{abstract}

Keywords: Knowledge Tracing · Network Embedding · Attention Mechanism - Student Assessment.

\section{Introduction}

Knowledge tracing (KT) is a modeling based on student behavior sequence. With the presence of KT, we can exactly grasp the students' mastery and the understanding of knowledge concepts. Accurate knowledge tracing enables us to grasp the current needs of students and to recommend questions accurately. This task means that students can be provided with resources according to their personal needs [1]. Knowledge tracing is the core and key to build an adaptive education system.

There exist two types of knowledge tracing models now: traditional knowledge tracing models and deep knowledge tracing models. Bayesian Knowledge Tracing (BKT) [2] is a very famous model among traditional knowledge tracing models. It is a Markov model with hidden variables. Recently, Deep Knowledge Tracing (DKT) [3] based on students' recent learning performance has shown high performance. It can model the relationship between complex knowledge concepts. 
Although knowledge tracing based on deep learning has made great progress, there are still some open issues. For example, the sparsity of students' behavior. Specifically, students usually use fragmented time to study, which only spend little time on the platform [4]. As a result, each student may only practice a small part of the test questions in system so that the mastery of a large part of knowledge is still unknown, which affects the knowledge tracing and limits the further application.

Our view of solving sparse problem depends on attribute information of questions. Specifically, the correct rate of the same student answering questions with similar difficulty or discrimination at the same knowledge concept is similar. Some existing work that study attributes of questions is primarily about mining them [5]. Other work try to use attributes of questions in knowledge tracing [6], but they do not specifically apply question attributes to deep knowledge tracing. By analyzing the various types of nodes and the various link relationships in the network, heterogeneous information network (HIN) [7] can accurately distinguish the different meanings in the network and mine more meaningful knowledge. Questions and their attributes can be expressed as a heterogeneous information network. Features of each question are thus captured by network embedding, which can guide us to better understand knowledge states.

In the sequence of student history questions, the contribution of each question to whether the next question can be answered correctly is different. For example, the next question mainly examines the mastery of integer multiplication, then the questions related to this concept in the past have a greater impact on the current state. Inspired by the attention mechanism used in machine translation where the mechanism can be used to capture the words corresponding to the original sentence and make the translation more accurate [8], we apply the attention mechanism to knowledge tracing problem to achieve better results.

In this paper, we focus on modeling the attribute information of questions with heterogeneous information network and generate meaningful question embeddings to model and predict students' knowledge states from a large number of learning logs. Our contributions are as follows:

- We use a heterogeneous information network to model questions and their attributes to learn the effective representations of questions.

- We propose Attention-based Knowledge Tracing with Heterogeneous Information Network Embedding to model students' current knowledge states.

- We evaluate our model and results show that our AKTHE model has better performance in knowledge tracing.

The rest of this paper is organized as follows. Section 2 gives a clear description on the problem definition. Section 3 is the detailed descriptions of proposed model. Experimental results on four datasets are shown in Section 4. Finally, we conclude the paper in Section 5. 


\section{Problem Definition}

Definition 1 (Question Difficulty.) Given a question, difficulty refers to the proportion or percentage of correct answers given by students in history, which is also known as the ease of question. The difficulty is defined as:

$$
D_{i}=k_{i} / N_{i},
$$

where $k_{i}$ is the number of students who answered question $i$ correctly, and $N_{i}$ is the total number of students who answered question $i$.

The value of question difficulty is between 0 and 1 . Instead of using difficulty value directly, we categorize every question into one of 10 difficulty levels:

$$
\text { Category }(i)= \begin{cases}\left\lfloor D_{i} \cdot c\right\rfloor, & \text { if }\left|N_{i}\right| \geq 4 \\ 5, & \text { else, }\end{cases}
$$

where $c=10$, the number of levels.

Definition 2 (Question Discrimination.) The discrimination refers to the ability of questions to distinguish academic performance between students. For simplicity, the following definition is used:

$$
D_{i}^{\prime}=D_{i}^{1}-D_{i}^{2}
$$

where $D_{i}^{\prime}$ is the discrimination of question $i$. We rank the total score of all students from high to low, the top $30 \%$ students is the high-level group and the last $30 \%$ students is the low-level group. $D_{i}^{1}$ and $D_{i}^{2}$ are the difficulty of question $i$ for high-level group and low-level group respectively using (1). Table 1 is a criterion for evaluating the discrimination of question.

Table 1: The division of question discrimination

\begin{tabular}{ccccc}
\hline Discrimination & Bad & General & Good & Nice \\
\hline Range & $(-1,0.19)$ & $(0.2,0.29)$ & $(0.3,0.39)$ & $(0.4,1)$ \\
\hline
\end{tabular}

Definition 3 (Heterogeneous Information Network (HIN) [7].) HIN is a directed graph $G=(V, E)$. Meanwhile, each node $v$ and each link $e$ has a type. Let $A$ and $R$ represent the sets of node type and link type respectively where $|A|+|R|>2$. For each node $v$, we have $\phi(v): V \rightarrow A$; for each link $e$, we have $\psi(e): E \rightarrow R$.

Definition 4 (Meta-Path [9].) A meta-path is defined as a path. The specific form is $A_{1} \stackrel{R_{1}}{\longrightarrow} A_{2} \stackrel{R_{2}}{\longrightarrow} \cdots A_{l-1} \stackrel{R_{l-1}}{\longrightarrow} A_{l}$. It represents a compound relationship between node types $A_{1}$ and $A_{l}$.

We express questions and their attributes using a HIN in Fig.1(a) where the node types are question, difficulty and discrimination. There exist two types of links between nodes: (i) the question-difficulty link, and (ii) the questiondiscrimination link. Nodes in the network can be connected into different kinds of 
meta-paths, e.g., "question-difficulty-question" $(Q D Q)$ and "question-discriminationquestion" $\left(Q D^{\prime} Q\right)$.

\section{Methodology}

We present Attention-based Knowledge Tracing with Heterogeneous Information Network Embedding, called AKTHE. Fig.1 represents the overall schematic illustration of AKTHE.

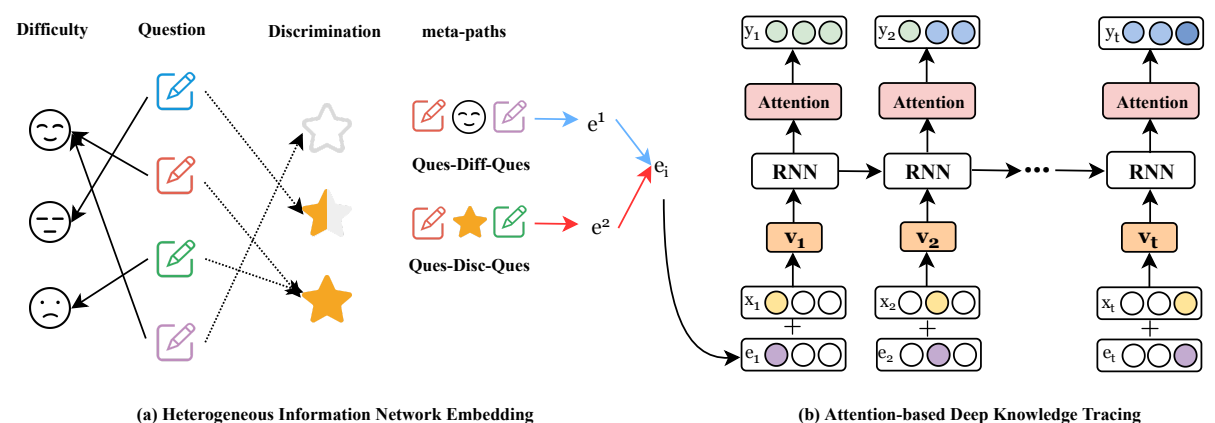

Fig. 1: The architecture of AKTHE.

\subsection{Heterogeneous Information Network Embedding}

Heterogeneous information network embedding (HINE) aims to generate meaningful node sequence. To this end, both DeepWalk [10] and node2vec [11] can be applied where a random walker traverses the heterogeneous information network from node to node along the edge between them.

Meta-Path Based Random Walk In the literature of HIN, meta-path [9] can depict the semantic concept accurately. Inspired by this, we use meta-path based random walk method. At step $t$, the walker moves from node $v_{t}$ to $v_{t+1}$, one of its neighbors, at the normalized probability $P\left(v_{t+1} \mid v_{t}\right)$. Note $v_{t}$ and $v_{t+1}$ can be of any type. Similarly, Given a HIN $G=(V, E)$ and a meta-path $\rho$ : $A_{1} \stackrel{R_{1}}{\longrightarrow} A_{2} \stackrel{R_{2}}{\longrightarrow} \cdots A_{t} \stackrel{R_{t}}{\longrightarrow} A_{t+1} \cdots \stackrel{R_{l-1}}{\longrightarrow} A_{l}$, the probability that the walker will take one step specified in the path is:

$$
P\left(v_{t+1} \mid v_{t}, \rho\right)=\left\{\begin{array}{cl}
\frac{1}{\left|N_{t+1}\left(v_{t}\right)\right|}, & \left(v_{t}, v_{t+1}\right) \in E, \phi\left(v_{t+1}\right)=A_{t+1} ; \\
0, & \text { otherwise }
\end{array}\right.
$$

where $v_{t}$ is the $t$ th node in random walk, as well as $N_{t+1}\left(v_{t}\right)$ is set of first-order neighbor nodes of $v_{t}$. 
Heterogeneous Skip-Gram For a given meta-path, we use a fixed-length window to obtain the neighborhood $N(v)$ for node $v$. Following node2vec [11], we use heterogeneous skip-gram to learn node embeddings for a $\operatorname{HIN} G=(V, E)$ by maximizing

$$
\max _{g} \sum_{v \in V} \log \mathrm{P}(N(v) \mid g(v)),
$$

where $g$ is a function to get node embedding.

Embedding Fusion For a node $v$, different embeddings on $O$ meta-paths can be represented as a set $\left\{e_{q}^{(l)}\right\}_{l=1}^{|O|}$, where $e_{q}^{(l)}$ is the embedding of question $q$ about the $l$ th meta-path. In our model, we only focus on the embeddings of questions, so we only select nodes with the type question.

For different representations of a question, we assume that the contribution of each meta-path to this question is equal. Then we use linear function as our fusion function:

$$
e_{q}=\frac{1}{|O|} \sum_{l=1}^{|O|}\left(\mathbf{M}^{(l)} e_{q}^{(l)}+\mathbf{b}^{(l)}\right),
$$

where $e_{q}$ is the final representation of question $q$.

\subsection{Attention-based Knowledge Tracing with HINE}

We use DKT model as the basic model because it is superior compared to BKT. In addition, we also added the attention mechanism to our model to learn better distribution of each question which may lead to more accurate prediction.

Input Formally, an interaction $\left\{q_{t}, a_{t}\right\}$ can be transformed into a vector $x_{t}$. We use $\boldsymbol{\delta}\left(q_{t}\right)$ to represent question $q_{t}$, which is a one-hot encoding. $x_{t}$ is a Cartesian product of $q_{t}$ and $a_{t}$. Before passing into LSTM, the embedding vector $x_{t}$ and $e_{t}$ in (6) are integrated by concatenation:

$$
\boldsymbol{v}_{\boldsymbol{t}}=x_{t} \oplus e_{t} .
$$

Attention Mechanism We employ the attention mechanism that concatenate the hidden layer and the historical questions $\left\{q_{1}, q_{2}, \ldots, q_{t}\right\}$, which scores the importance of each question in the history for new question $q_{t+1}$ :

$$
u_{t}^{i}=\mathbf{v}^{T} \tanh \left(\mathbf{W}_{1} h_{t}+\mathbf{W}_{2} \boldsymbol{\delta}\left(q_{i}\right)\right)
$$

where $\boldsymbol{\delta}\left(q_{i}\right)$ is the one-hot encoding of question $q_{i}$ in historical questions and $h_{t}$ is hidden state.

Then attention state can be represented as the weighted sum of all questions in this step by using attention scores as weights. That is,

$$
s_{t}=\sum_{i=1}^{t} a_{t}^{i} u_{t}^{i}, a_{t}^{i}=\frac{\exp \left(\mathbf{W}_{a} u_{t}^{i}\right)}{\sum_{j=1}^{t} \exp \left(\mathbf{W}_{a} u_{t}^{j}\right)} .
$$


Output Finally, based on the updated hidden state $h_{t}$ and attention state $s_{t}$, we can get the probability of answering each question correctly:

$$
\mathbf{y}_{t}=\sigma\left(\mathbf{W}\left(s_{t} \oplus h_{t}\right)+\mathbf{b}\right) .
$$

Optimization The purpose of AKTHK is to predict next interaction performance. In this paper, we use the cross-entropy loss function to learn the parameters:

$$
\mathcal{L}=-\sum_{t}\left(a_{t+1} \log \left(\mathbf{y}_{t}^{\mathrm{T}} \boldsymbol{\delta}\left(q_{t+1}\right)\right)+\left(1-a_{t+1}\right) \log \left(1-\mathrm{y}_{t}^{\mathrm{T}} \boldsymbol{\delta}\left(q_{t+1}\right)\right)\right),
$$

where $\boldsymbol{\delta}\left(q_{t+1}\right)$ and $a_{t+1}$ are the one-hot encoding and the true label of next question $q_{t+1}$.

\section{Experiments}

In this section, we demonstrate the effectiveness of our proposed AKTHE model by comparing it with baselines on four datasets.

\subsection{Experimental Setup}

Datasets For our experiments, we test on four public datasets: ASSISTments2009, ASSISTments2015, Statics2011 and Synthetic-5. The statistical information of datasets is reported in Table 2 .

Table 2: Dataset statistics.

\begin{tabular}{cccc}
\hline Datasets & Students & Exercises & Records \\
\hline ASSISTments2009 & 4,151 & 110 & 325,637 \\
ASSISTments2015 & 19,840 & 100 & 683,801 \\
Synthetic-5 & 4,000 & 50 & 200,000 \\
Statics2011 & 333 & 1,223 & 189,297 \\
\hline
\end{tabular}

Baselines To demonstrate performance of our AKTHE model, we compare with multiple baselines in each dataset:

Bayesian Knowledge Tracing (BKT) : In BKT [2] model, a hidden variable about knowledge states of students is proposed. BKT is to track changes in state of students' knowledge.

Deep Knowledge Tracing (DKT): DKT [3] is the most widely used knowledge tracing model. Its performance on a variety of open datasets is basically better than traditional models.

Dynamic Key-Value Memory Networks (DKVMN): DKVMN [12] is a state-of-the-art KT model. This model uses a static matrix key to store all knowledge concepts and a dynamic matrix value to store and update learners' mastery of concepts. 


\subsection{Performance Analysis}

The performance of knowledge tracing is customarily evaluated by area under the curve (AUC). The higher the AUC, the better the model performance. The AUC Results of models on all datasets are shown in Table 3.

Table 3: The AUC results on all datasets.

\begin{tabular}{lcccc}
\hline Model & ASSISTments2009 & ASSISTments2015 & Synthetic-5 & Statics2011 \\
\hline BKT & 0.623 & 0.631 & 0.650 & 0.730 \\
DKT & 0.805 & 0.725 & 0.803 & 0.802 \\
DKVMN & 0.816 & 0.727 & 0.827 & 0.828 \\
\hline DKT+Attention & 0.827 & 0.739 & 0.835 & 0.834 \\
DKT+HINE & 0.818 & 0.735 & 0.829 & 0.832 \\
AKTHE & $\mathbf{0 . 8 3 1}$ & $\mathbf{0 . 7 4 1}$ & $\mathbf{0 . 8 3 7}$ & $\mathbf{0 . 8 3 8}$ \\
\hline
\end{tabular}

From Table 3, we can get two points of view. Firstly, compared with the benchmark models, our model has higher AUC, which means a better prediction performance. Concretely speaking, the average AUC obtained by AKTHE on two ASSISTments datasets is $1.8 \%$ higher than the most advanced model DKVMN. Compared with DKVMN, the average improvement rates are $1.1 \%$ in synthetic5 and statics2011 datasets. Secondly, models based on neural network perform better than traditional model on four datasets, which means that the neural network can capture more useful information.

The results of ablation experiments show that the combination of HINE or attention with DKT can all improve the prediction effect. Adding attention to DKT contributes to the better results, which means that the attention mechanism provides more useful information.

\section{Conclusion}

In this paper, we mainly focus on exploring the attribute information of questions in knowledge tracing. We describe questions and their attributes with a heterogeneous information network, which is desirable for this application. With the help of heterogeneous network embedding, the learned node embeddings can be integrated into deep knowledge tracing model. We also add the attention mechanism to deep knowledge tracing model. On four datasets for knowledge tracing, our model has better performance than BKT, DKT and DKVMN.

In the future, we intend to introduce the relationship between concepts contained in the questions by using the method of graph embedding. 


\section{Acknowledgements}

This research was supported by NSFC (Grants No. 61877051), and Natural Science Foundation Project of CQ, China (Grants No. cstc2018jscx-msyb1042, and cstc2018jscx-msybX0273). Li Li is the corresponding author for the paper.

\section{References}

1. Xu Daomin and Dong Mingchui. Appropriate learning resource recommendation in intelligent web-based educational system. In ISDEA, pages 169-173. IEEE, 2013.

2. Albert T Corbett and John R Anderson. Knowledge tracing: Modeling the acquisition of procedural knowledge. UMUAI, 4(4):253-278, 1994.

3. Chris Piech, Jonathan Bassen, Jonathan Huang, Surya Ganguli, Mehran Sahami, Leonidas J Guibas, and Jascha Sohl-Dickstein. Deep knowledge tracing. In NIPS, pages 505-513, 2015.

4. Jiayin Lin, Geng Sun, Tingru Cui, Jun Shen, Dongming Xu, Ghassan Beydoun, Ping Yu, David E Pritchard, Li Li, and Shiping Chen. From ideal to reality: segmentation, annotation, and recommendation, the vital trajectory of intelligent micro learning. World Wide Web, pages 1-21, 2019.

5. Sankaran Narayanan, Vamsi Sai Kommuri, N. Sethu Subramanian, Kamal Bijlani, and Nandu C. Nair. Unsupervised learning of question difficulty levels using assessment responses. In ICCSA, pages 543-552, 2017.

6. José González-Brenes, Yun Huang, and Peter Brusilovsky. General features in knowledge tracing to model multiple subskills, temporal item response theory, and expert knowledge. In EDM, pages 84-91. University of Pittsburgh, 2014.

7. Chuan Shi, Yitong Li, Jiawei Zhang, Yizhou Sun, and S Yu Philip. A survey of heterogeneous information network analysis. TKDE, 29(1):17-37, 2016.

8. Dzmitry Bahdanau, Kyunghyun Cho, and Yoshua Bengio. Neural machine translation by jointly learning to align and translate. arXiv preprint arXiv:1409.0473, 2014.

9. Yizhou Sun, Jiawei Han, Xifeng Yan, Philip S Yu, and Tianyi Wu. Pathsim: Meta path-based top-k similarity search in heterogeneous information networks. PVLDB, 4(11):992-1003, 2011.

10. Bryan Perozzi, Rami Al-Rfou, and Steven Skiena. Deepwalk: Online learning of social representations. In KDD, pages 701-710. ACM, 2014.

11. Aditya Grover and Jure Leskovec. node2vec: Scalable feature learning for networks. In $K D D$, pages 855-864. ACM, 2016.

12. Jiani Zhang, Xingjian Shi, Irwin King, and Dit-Yan Yeung. Dynamic key-value memory networks for knowledge tracing. In $W W W$, pages $765-774$. International World Wide Web Conferences Steering Committee, 2017. 